\title{
On power and non-power asymptotic behavior of positive solutions to Emden-Fowler type higher-order equations
}

Irina Astashova*

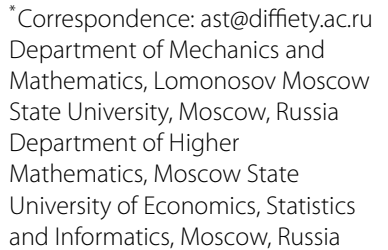

\section{Abstract}

For the equation

$$
y^{(n)}=y^{k}, \quad k>1, n=12,13,14,
$$

the existence of positive solutions with non-power asymptotic behavior is proved, namely

$$
y=\left(x^{*}-x\right)^{-\alpha} h\left(\log \left(x^{*}-x\right)\right), \quad \alpha=\frac{n}{k-1}, x<x^{*},
$$

where $x^{*}$ is an arbitrary point, $h$ is a positive periodic non-constant function on $\mathbf{R}$.

To prove this result, the Hopf bifurcation theorem is used.

Keywords: asymptotic behavior; Emden-Fowler higher-order equations

\section{Introduction}

For the equation

$$
y^{(n)}=p\left(x, y, y^{\prime}, \ldots, y^{(n-1)}\right)|y|^{k} \operatorname{sgn} y, \quad n \geq 2, k>1,
$$

Kiguradze posed the problem on the asymptotic behavior of its positive solutions such that

$$
\lim _{x \rightarrow x^{*}-0} y(x)=\infty
$$

He found an asymptotic formula for these solutions to (1) with $n=2$ (see [1]) and supposed all such solutions to have power asymptotic behavior for other $n$, too. The problem was solved for $n=3$ and $n=4$ [2]. For these $n$, it was proved that all such solutions behave as

$$
y(x)=C\left(x^{*}-x\right)^{-\alpha}(1+o(1)), \quad x \rightarrow x^{*}-0,
$$

2013 Astashova; licensee Springer. This is an Open Access article distributed under the terms of the Creative Commons Attribution License (http://creativecommons.org/licenses/by/2.0), which permits unrestricted use, distribution, and reproduction in any medium, provided the original work is properly cited. 
with

$$
\alpha=\frac{n}{k-1}, \quad C=\left(\frac{\alpha(\alpha+1) \cdots(\alpha+n-1)}{p_{0}}\right)^{\frac{1}{k-1}},
$$

$p_{0}=$ const $>0$ - is a limit of $p\left(x, y_{0}, \ldots, y_{n-1}\right)$ as $x \rightarrow x^{*}-0, y_{0} \rightarrow \infty, \ldots, y_{n-1} \rightarrow \infty$.

So, the hypothesis of Kiguradze was confirmed in this case.

The existence of solutions satisfying (3) was proved for arbitrary $n \geq 2$. For $2 \leq n \leq 11$, an $(n-1)$-parametric family of such solutions to equation (1) was proved to exist (see [2], [3], Ch.I (5.1)).

For the equation

$$
y^{(n)}=y^{k}, \quad k>1
$$

a negative answer to the conjecture of Kiguradze for large $n$ was obtained. It was proved [4] that for any $N$ and $K>1$, there exist an integer $n>N$ and $k \in \mathbf{R}, 1<k<K$, such that equation (5) has a solution

$$
y=\left(x^{*}-x\right)^{-\alpha} h\left(\log \left(x^{*}-x\right)\right),
$$

where $\alpha$ is defined by (4), $h$ is a positive periodic non-constant function on $\mathbf{R}$.

Still, it was not clear how large $n$ should be for the existence of that type of solutions.

\section{Preliminary results}

Suppose the following conditions hold:

(A) The continuous positive function $p\left(x, y_{0}, \ldots, y_{n-1}\right)$ has a limit $p_{0}=$ const $>0$ as $x \rightarrow$ $x^{*}-0, y_{0} \rightarrow \infty, \ldots, y_{n-1} \rightarrow \infty$, and for some $\gamma>0$, it holds

$$
p\left(x, y_{0}, \ldots, y_{n-1}\right)-p_{0}=O\left(\left(x^{*}-x\right)^{\gamma}+\sum_{j=0}^{n-1} y_{j}^{-\gamma}\right) .
$$

(B) For some $K_{1}>0$ and $\mu>0$ in a neighborhood of $x^{*}$ for sufficiently large $y_{0}, \ldots, y_{n-1}$, $z_{0}, \ldots, z_{n-1}$, it holds

$$
\left|p\left(x, y_{0}, \ldots, y_{n-1}\right)-p\left(x, z_{0}, \ldots, z_{n-1}\right)\right| \leq K_{1} \max _{j}\left|y_{j}^{-\mu}-z_{j}^{-\mu}\right|
$$

Then equation (1) can be transformed (see [2] or [3], Ch.I(5.1)) by using the substitution

$$
x^{*}-x=e^{-t}, \quad y=(C+v) e^{\alpha t},
$$

where $C$ and $\alpha$ are defined by (4). The derivatives $y^{(j)}, j=0,1, \ldots, n-1$, become

$$
e^{(\alpha+j) t} \cdot L_{j}\left(v, v^{\prime}, \ldots, v^{(j)}\right)
$$

where $v^{(j)}=\frac{d^{j} v}{d t^{j}}$ and $L_{j}$ is a linear function with

$$
L_{j}(0,0, \ldots, 0)=C \alpha(\alpha+1) \cdots(\alpha+j-1) \neq 0
$$

and the coefficient of $v^{(j)}$ equal to 1 . 
Thus (1) is transformed into

$$
e^{(\alpha+n) t} \cdot L_{n}\left(v, v^{\prime}, \ldots, v^{(n)}\right)=(C+v)^{k} e^{\alpha k t} \tilde{p}\left(t, v, v^{\prime}, \ldots, v^{(n-1)}\right)
$$

where the function $\tilde{p}\left(t, v_{0}, \ldots, v_{n-1}\right)$ is obtained from $p\left(x, y_{0}, \ldots, y_{n-1}\right)$ with $x, y_{0}, \ldots, y_{n-1}$ properly expressed in terms of $t, v_{0}, \ldots, v_{n-1}$. This function tends to $p_{0}$ as $t \rightarrow \infty, v \rightarrow$ $0, \ldots, v^{(n-1)} \rightarrow 0$.

Due to condition (8) for the function $p\left(x, y_{0}, \ldots, y_{n-1}\right)$, we obtain the following inequalities for sufficiently large $t$ and sufficiently small $v_{0}, \ldots, v_{n-1}, w_{0}, \ldots, w_{n-1}$ :

$$
\begin{aligned}
& \left|\tilde{p}\left(t, v_{0}, \ldots, v_{n-1}\right)-\tilde{p}\left(t, w_{0}, \ldots, w_{n-1}\right)\right| \\
& \quad \leq K_{1} \max _{j} e^{-\mu(\alpha+j) t}\left|L_{j}^{-\mu}\left(v_{0}, \ldots, v_{n-1}\right)-L_{j}^{-\mu}\left(w_{0}, \ldots, w_{n-1}\right)\right| .
\end{aligned}
$$

Since $L_{j}(0,0, \ldots, 0) \neq 0$, the function $L_{j}^{-\mu}$ is a $C^{\infty}$ one in a neighborhood of 0 and

$$
\left|\tilde{p}\left(t, v_{0}, \ldots, v_{n-1}\right)-\tilde{p}\left(t, w_{0}, \ldots, w_{n-1}\right)\right| \leq K_{2} e^{-\mu \alpha t} \max _{j}\left|v_{j}-w_{j}\right|
$$

for some $K_{2}>0$.

Solving (10) for $v^{(n)}$ and using formulae (4), we obtain the equation

$$
v^{(n)}=(C+v)^{k} \tilde{p}\left(t, v, v^{\prime}, \ldots, v^{(n-1)}\right)-p_{0} C^{k}-\sum_{j=0}^{n-1} a_{j} v^{(j)},
$$

where $a_{j}$ are the coefficients of the linear function $L_{n}$. Equation (11) can be written as

$$
v^{(n)}=k C^{k-1} p_{0} v-\sum_{j=0}^{n-1} a_{j} v^{(j)}+f(v)+g\left(t, v, v^{\prime}, \ldots, v^{(n-1)}\right),
$$

where

$$
\begin{aligned}
& \begin{array}{l}
f(v)=p_{o}\left((C+v)^{k}-C^{k}-k C^{k-1} v\right)=O\left(v^{2}\right) \quad \text { as } v \rightarrow 0, \\
f^{\prime}(v)=O(v) \quad \text { as } v \rightarrow 0, \\
g\left(t, v_{0}, \ldots, v_{n-1}\right)=\left(C+v_{0}\right)^{k}\left(\tilde{p}\left(t, v_{0}, \ldots, v_{n-1}\right)-p_{0}\right) \\
=O\left(e^{-\gamma t}+\sum_{j=0}^{n-1} e^{-\gamma(\alpha+j) t}\right)=O(\exp (-\gamma \min (\alpha, 1) t)) \\
\quad \text { as } t \rightarrow \infty, v_{0} \rightarrow 0, \ldots, v_{n-1} \rightarrow 0 .
\end{array}
\end{aligned}
$$

Besides, for sufficiently large $t$ and sufficiently small $v_{0}, \ldots, v_{n-1}, w_{0}, \ldots, w_{n-1}$, it holds

$$
\begin{aligned}
\mid g(t, & \left.v_{0}, \ldots, v_{n-1}\right)-g\left(t, w_{0}, \ldots, w_{n-1}\right) \mid \\
\leq & \left|\left(C+v_{0}\right)^{k}-\left(C+w_{0}\right)^{k}\right| \cdot\left|\tilde{p}\left(t, v_{0}, \ldots, v_{n-1}\right)-p_{0}\right| \\
& +\left(C+w_{0}\right)^{k}\left|\tilde{p}\left(t, v_{0}, \ldots, v_{n-1}\right)-\tilde{p}\left(t, w_{0}, \ldots, w_{n-1}\right)\right| \\
\leq & K_{3} \max _{j}\left|w_{j}-v_{j}\right| e^{-\min (\gamma, \mu) \cdot \min (\alpha, 1) t} .
\end{aligned}
$$


Suppose that $V$ is the vector with coordinates $V_{j}=v^{(j)}, j=0, \ldots, n-1$. Then equation (12) can be written as

$$
\frac{d V}{d t}=A V+F(V)+G(t, V)
$$

where $A$ is a constant $n \times n$ matrix

$$
A=\left(\begin{array}{cccccc}
0 & 1 & 0 & 0 & \cdots & 0 \\
0 & 0 & 1 & 0 & \cdots & 0 \\
0 & 0 & 0 & 1 & \cdots & 0 \\
\cdot & \cdot & \cdot & \cdot & \cdots & \cdot \\
0 & 0 & 0 & 0 & \cdots & 1 \\
-\tilde{a}_{0} & -a_{1} & -a_{2} & -a_{3} & \cdots & -a_{n-1}
\end{array}\right),
$$

with

$$
\begin{aligned}
-\tilde{a}_{0} & =a_{0}-k c^{k-1} p_{0}=a_{0}-k \alpha(\alpha+1) \cdots(\alpha+n-1) \\
& =a_{0}-(\alpha+1) \cdots(\alpha+n-1)(\alpha+n)
\end{aligned}
$$

and eigenvalues satisfying the equation

$$
\begin{aligned}
0 & =\operatorname{det}(A-\lambda E)=(-1)^{n+1}\left(-\tilde{a}_{0}-a_{1} \lambda-\cdots-a_{n-1} \lambda^{n-1}-\lambda^{n}\right) \\
& =(-1)^{n+1}((\alpha+1)(\alpha+2) \cdots(\alpha+n)-(\lambda+\alpha) \cdots(\lambda+\alpha+n-1)),
\end{aligned}
$$

which is equivalent to

$$
\prod_{j=0}^{n-1}(\lambda+\alpha+j)=\prod_{j=0}^{n-1}(1+\alpha+j)
$$

The mappings $F: \mathbf{R}^{n} \rightarrow \mathbf{R}^{n}$ and $G: \mathbf{R} \times \mathbf{R}^{n} \rightarrow \mathbf{R}^{n}$ satisfy the following estimates as $t \rightarrow \infty$ :

$$
\left\{\begin{array}{l}
\|F(V)\|=O\left(\|V\|^{2}\right) \\
\left\|F_{V}^{\prime}(V)\right\|=O(\|V\|) \\
\|G(t, V)\|=O\left(e^{-2 \beta t}\right) \\
\|G(t, V)-G(t, W)\| \leq K\|V-W\| e^{-2 \beta t}
\end{array}\right.
$$

with some constants $\beta>0, K>0$.

Lemma 1 [3] Suppose that (15) holds and $A$ is an arbitrary constant $n \times n$ matrix. Then there exists a solution $V(t)$ to equation (13) tending to zero as $t \rightarrow \infty$.

Lemma 2 [3] Let the conditions of Lemma 1 hold. If equation (14) has m roots with negative real part, then there exists an m-parametric family of solutions $V(t)$ to equation (13) tending to zero as $t \rightarrow \infty$. 
If equation (13) has a solution $V(t)$ tending to 0 as $t \rightarrow \infty$ and $V_{0}(t)$ is its first coordinate, then the function

$$
y(x)=\left(V_{0}\left(-\log \left(x^{*}-x\right)\right)+C\right) \cdot\left(x^{*}-x\right)^{-\alpha}
$$

with $C$ and $\alpha$ defined by (4) is a solution to (1) such that (2) and (3) hold.

Theorem $1[2,3]$ Suppose that conditions (A) and (B) are satisfied. Then for such $x^{*}$ there exists a solution to (1) with power asymptotic behavior (3).

Investigating the signs of the real parts of the roots of equation (14), by the RouthHurwitz criterion, we can prove the following theorem.

Theorem 2 [2,3] Suppose that $3 \leq n \leq 11$ and conditions (A) and (B) are satisfied. Then there exists an (n-1)-parametric family of solutions to equation (1) with power asymptotic behavior (3).

Theorem $3[2,3,5]$ Suppose that $n=3$ or $n=4$ in equation (1), the continuous positive function $p\left(x, y_{0}, \ldots, y_{n-1}\right)$ is Lipschitz continuous in $y_{0}, \ldots, y_{n-1}$ and has a limit $p_{0}>0$ as $x \rightarrow x^{*}-0, y_{0} \rightarrow \infty, \ldots, y_{n-1} \rightarrow \infty$. Then any positive solution to this equation with a vertical asymptote $x=x^{*}$ has asymptotic behavior (3).

To prove the main results of this article, we use the Hopf bifurcation theorem [6].

Theorem (Hopf) Consider the $\alpha$-parameterized dynamical system $\dot{x}=L_{\alpha} x+Q_{\alpha}(x)$ in a neighborhood of $0 \in \mathbf{R}^{n}$ with linear operators $L_{\alpha}$ and smooth enough functions $Q_{\alpha}(x)=$ $O\left(|x|^{2}\right)$ as $x \rightarrow 0$. Let $\lambda_{\alpha}$ and $\bar{\lambda}_{\alpha}$ be simple complex conjugated eigenvalues of the operators $L_{\alpha}$. Suppose that $\operatorname{Re} \lambda_{\tilde{\alpha}}=\operatorname{Re} \bar{\lambda}_{\tilde{\alpha}}=0$ for some $\tilde{\alpha}$ and the operator $L_{\tilde{\alpha}}$ has no other eigenvalues with zero real part.

If $\operatorname{Re} \frac{d \lambda_{\alpha}}{d \alpha}(\tilde{\alpha}) \neq 0$, then there exist continuous mappings $\varepsilon \mapsto \alpha(\varepsilon) \in \mathbf{R}, \varepsilon \mapsto T(\varepsilon) \in \mathbf{R}$, and $\varepsilon \mapsto b(\varepsilon) \in \mathbf{R}^{n}$ defined in a neighborhood of 0 and such that $\alpha(0)=\tilde{\alpha}, T(0)=2 \pi / \operatorname{Im} \lambda_{\tilde{\alpha}}$, $b(0)=0, b(\varepsilon) \neq 0$ for $\varepsilon \neq 0$, and the solutions to the problems

$$
\dot{x}=L_{\alpha(\varepsilon)} x+Q_{\alpha(\varepsilon)}(x), \quad x(0)=b(\varepsilon)
$$

are $T(\varepsilon)$-periodic and non-constant.

\section{Main results}

In this section, the result about the existence of solutions with non-power asymptotic behavior is proved for equation (5) with $n=12,13,14$.

Theorem 4 For $n=12,13,14$, there exists $k>1$ such that equation (5) has a solution $y(x)$ with

$$
\begin{gathered}
y^{(j)}(x)=\left(x^{*}-x\right)^{-\alpha-j} h_{j}\left(\log \left(x^{*}-x\right)\right), \\
j=0,1, \ldots, n-1,
\end{gathered}
$$

where $\alpha$ is defined by (4) and $h_{j}$ are periodic positive non-constant functions on $\mathbf{R}$. 
Proof To apply the Hopf bifurcation theorem, we investigate equation (13) with $G(t, V) \equiv$ 0 corresponding to the case of the constant function $p$ and the roots of the algebraic equation (14). $F$ is a vector function with all zero components $F(V)=\left(0, \ldots, 0, F_{n-1}(V)\right)$, $V=\left(V_{0}, \ldots, V_{n-1}\right)$, and

$$
\begin{aligned}
& F_{n-1}(V)=\left(\left(C+V_{0}\right)^{k}-C^{k}-k C^{k-1} V_{0}\right)=O\left(V_{0}^{2}\right), \quad V_{0} \rightarrow 0, \\
& \frac{d}{d V} F_{n-1}(V)=O\left(\left|V_{0}\right|\right), \quad V_{0} \rightarrow 0 .
\end{aligned}
$$

If equation (14) has a pair of pure imaginary roots, we have to check other conditions of this theorem and then apply it.

Proposition 1 For any integer $n>11$, there exist $\alpha>0$ and $q>0$ such that

$$
\prod_{j=0}^{n-1}(q i+\alpha+j)=\prod_{j=0}^{n-1}(1+\alpha+j)
$$

with $i^{2}=-1$.

Remark 1 In the particular case $n=12$, this result was obtained by Vyun [7].

Proof Consider the positive functions $\rho_{n}(\alpha)$ and $\sigma_{n}(\alpha)$ defined for all $\alpha>0$ via the equations

$$
\prod_{j=0}^{n-1}\left(\rho_{n}(\alpha)^{2}+(\alpha+j)^{2}\right)=\prod_{j=0}^{n-1}(1+\alpha+j)^{2}
$$

and

$$
\sum_{j=0}^{n-1} \arg \left(\sigma_{n}(\alpha) i+\alpha+j\right)=2 \pi
$$

supposing $\arg z \in[0,2 \pi)$ for all $z \in \mathbb{C} \backslash\{0\}$.

First, we prove the functions to be well defined for all $\alpha>0$.

The product $\prod_{j=0}^{n-1}\left(q^{2}+(\alpha+j)^{2}\right)$ is continuous and strictly increasing as a function of $q>0$.

It tends to $\prod_{j=0}^{n-1}(\alpha+j)^{2}<\prod_{j=0}^{n-1}(1+\alpha+j)^{2}$ as $q \rightarrow 0$ and to $+\infty$ as $q \rightarrow+\infty$. Hence, for any $\alpha>0$, there exists a unique $q>0$ such that $\prod_{j=0}^{n-1}\left(q^{2}+(\alpha+j)^{2}\right)=\prod_{j=0}^{n-1}(1+\alpha+j)^{2}$.

In the same way, for any $\alpha>0$, the sum $\sum_{j=0}^{n-1} \arg (q i+\alpha+j)$ is a continuous function of $q>0$ strictly increasing from 0 to $\frac{\pi n}{2}>2 \pi$. So, there exists a unique $q>0$ such that the sum is equal to $2 \pi$.

Since both the product and the sum considered are $C^{1}$-functions with positive partial derivative in $q>0$, the implicit function theorem provides both $\rho_{n}(\alpha)$ and $\sigma_{n}(\alpha)$ to be $C^{1}$-functions, too.

Now it is sufficient to prove the existence of $\alpha>0$ such that $\rho_{n}(\alpha)$ and $\sigma_{n}(\alpha)$ are equal to the same value $q$, which makes the two sides of (16) be equal.

Compare the functions $\rho_{n}(\alpha)$ and $\sigma_{n}(\alpha)$ near the boundaries of their common domain. 
Equation (17) defining the function $\rho_{n}(\alpha)$ may be written as

$$
\prod_{j=0}^{n-1}\left(1+\frac{2 j}{\alpha}+\frac{j^{2}}{\alpha^{2}}+\left(\frac{\rho_{n}(\alpha)}{\alpha}\right)^{2}\right)=\prod_{j=0}^{n-1}\left(1+\frac{j+1}{\alpha}\right)^{2} .
$$

This shows that $\frac{\rho_{n}(\alpha)}{\alpha} \rightarrow 0$ as $\alpha \rightarrow+\infty$.

Equation (18) defining the function $\sigma_{n}(\alpha)$ may be written as

$$
\sum_{j=0}^{n-1} \arctan \frac{\frac{\sigma_{n}(\alpha)}{\alpha}}{1+\frac{j}{\alpha}}=2 \pi
$$

This shows that $\frac{\sigma_{n}(\alpha)}{\alpha} \rightarrow \tan \frac{2 \pi}{n}>0$ as $\alpha \rightarrow+\infty$. Thus, $\rho_{n}(\alpha)<\sigma_{n}(\alpha)$ for sufficiently large $\alpha$.

Now, to prove Proposition 1 , it is sufficient to show that $\rho_{n}(\alpha)>\sigma_{n}(\alpha)$ for sufficiently small $\alpha$. To compare the functions $\rho_{n}(\alpha)$ and $\sigma_{n}(\alpha)$ for small $\alpha>0$, we need some lemmas.

Lemma 3 For all $\alpha>0$, it holds $\rho_{n}(\alpha)^{2}<2(\alpha+n)-1$.

Proof Suppose that $\rho_{n}(\alpha)^{2} \geq 2(\alpha+n)-1$ for some $\alpha>0$. Then

$$
\begin{aligned}
\prod_{j=0}^{n-1}\left(\rho_{n}(\alpha)^{2}+(\alpha+j)^{2}\right) & \geq \prod_{j=0}^{n-1}\left(2(\alpha+n)-1+(\alpha+j)^{2}\right) \\
& >\prod_{j=0}^{n-1}\left(2(\alpha+j+1)-1+(\alpha+j)^{2}\right)=\prod_{j=0}^{n-1}(1+(\alpha+j))^{2}
\end{aligned}
$$

This contradiction with the definition of $\rho_{n}(\alpha)$ completes the proof of Lemma 3 .

Lemma 4 For all $\alpha>0$, it holds $\rho_{n+1}(\alpha)>\rho_{n}(\alpha)$.

Proof According to the definition of $\rho_{n}(\alpha)$ by (17) and Lemma 3, we have

$$
\begin{aligned}
\prod_{j=0}^{n}\left(\rho_{n}(\alpha)^{2}+(\alpha+j)^{2}\right) & =\prod_{j=0}^{n-1}(1+\alpha+j)^{2} \cdot\left(\rho_{n}(\alpha)^{2}+(\alpha+n)^{2}\right) \\
& <\prod_{j=0}^{n-1}(1+\alpha+j)^{2} \cdot\left(2(\alpha+n)-1+(\alpha+n)^{2}\right)<\prod_{j=0}^{n}(1+\alpha+j)^{2} .
\end{aligned}
$$

In order to make the first and the last products be equal, we have to replace $\rho_{n}(\alpha)$ in the first one by a greater value. This means that $\rho_{n+1}(\alpha)>\rho_{n}(\alpha)$ and Lemma 4 is proved.

Lemma 5 For all $\alpha>0$, it holds $\sigma_{n+1}(\alpha)<\sigma_{n}(\alpha)$.

Proof According to the definition of $\sigma_{n}(\alpha)$ by (18), we have

$$
\sum_{j=0}^{n} \arg \left(\sigma_{n}(\alpha) i+\alpha+j\right)=2 \pi+\arg \left(\sigma_{n}(\alpha) i+\alpha+n\right)>2 \pi .
$$


In order to make the sum equal $2 \pi$, we have to replace $\sigma_{n}(\alpha)$ by a smaller value. So, $\sigma_{n+1}(\alpha)<\sigma_{n}(\alpha)$ and Lemma 5 is proved.

Due to Lemmas 3, 4, 5 proved, it is sufficient now for the proof of Proposition 1 to show that $\rho_{12}(\alpha)>\sigma_{12}(\alpha)$ for sufficiently small $\alpha>0$.

Lemma 6 It holds $\rho_{12}(\alpha)>2$ for all sufficiently small $\alpha>0$.

Proof Straightforward exact calculations show that

$$
\lim _{\alpha \rightarrow 0} \prod_{j=0}^{11}\left(2^{2}+(\alpha+j)^{2}\right)=\prod_{j=0}^{11}\left(4+j^{2}\right)=192,175,659,520,000,000<2 \cdot 10^{17}
$$

and

$$
\lim _{\alpha \rightarrow 0} \prod_{j=0}^{11}(1+\alpha+j)^{2}=(12 !)^{2}=229,442,532,802,560,000>2 \cdot 10^{17} .
$$

So, for sufficiently small $\alpha>0$, we have

$$
\prod_{j=0}^{11}\left(2^{2}+(\alpha+j)^{2}\right)<2 \cdot 10^{17}<\prod_{j=0}^{11}(1+\alpha+j)^{2}
$$

Hence, for these $\alpha$, in order to avoid contradiction with the definition of $\rho_{12}(\alpha)$, the inequality $\rho_{12}(\alpha)^{2}>2^{2}$ is necessary. Lemma 6 is proved.

Lemma 7 It holds $\sigma_{12}(\alpha)<2$ for sufficiently small $\alpha>0$.

Proof Consider the limit

$$
\begin{aligned}
\lim _{\alpha \rightarrow 0} \sum_{j=0}^{11} \arg (2 i+\alpha+j) \\
=\arg 2 i+\arctan 2+\arctan 1+\arctan \frac{2}{3}+\arctan \frac{1}{2}+\sum_{j=5}^{11} \arctan \frac{2}{j} \\
=\frac{5 \pi}{4}+\arctan \frac{2}{3}+\sum_{j=5} \arctan \frac{2}{j} \\
=\frac{5 \pi}{4}+\arctan \frac{\frac{2}{3}+\frac{2}{5}}{1-\frac{2}{3} \cdot \frac{2}{5}}+\arctan \frac{\frac{2}{6}+\frac{2}{7}}{1-\frac{2}{6} \cdot \frac{2}{7}}+\arctan \frac{\frac{2}{8}+\frac{2}{9}}{1-\frac{2}{8} \cdot \frac{2}{9}}+\arctan \frac{\frac{2}{10}+\frac{2}{11}}{1-\frac{2}{10} \cdot \frac{2}{11}} \\
=\frac{5 \pi}{4}+\arctan \frac{16}{11}+\arctan \frac{13}{19}+\arctan \frac{1}{2}+\arctan \frac{21}{53} \\
=\frac{5 \pi}{4}+\arctan \frac{\frac{16}{11}+\frac{13}{19}}{1-\frac{16}{11} \cdot \frac{13}{19}}+\arctan \frac{\frac{1}{2}+\frac{21}{53}}{1-\frac{1}{2} \cdot \frac{21}{53}}=\frac{5 \pi}{4}+\arctan 447+\arctan \frac{19}{17} .
\end{aligned}
$$


Note that

$$
\tan \left(\arctan 447+\arctan \frac{19}{17}\right)=\frac{447+\frac{19}{17}}{1-447 \cdot \frac{19}{17}}=-\frac{3,809}{4,238}
$$

Hence, $\arctan 447+\arctan \frac{19}{17}>\frac{3 \pi}{4}$ and $\sum_{j=0}^{11} \arg (2 i+\alpha+j)>2 \pi$ for sufficiently small $\alpha>0$. Thus, for these $\alpha$, we have $\sigma_{12}(\alpha)<2$, which completes the proof of Lemma 7 .

Now Proposition 1 is also proved.

Proposition 2 For any $\alpha>0$ and any integer $n>1$, all roots $\lambda \in \mathbb{C}$ to equation (14) are simple.

Proof Since we consider a polynomial equation of degree $n$, it is sufficient to prove the existence of $n$ different roots to (14). We will show that for any integer $m$ such that $-n<$ $m \leq n$, there exists $\mu_{m} \in \mathbb{C}$ satisfying

$$
\prod_{j=0}^{n-1}\left|\mu_{m}+j\right|=\prod_{j=0}^{n-1}(1+\alpha+j)
$$

and

$$
\sum_{j=0}^{n-1} \arg \left(\mu_{m}+j\right)=m \pi
$$

with $\arg z$ denoting the principal value of the argument lying in the open-closed interval $(-\pi, \pi]$. Surely, all these $2 n$ complex numbers $\mu_{m}$ are different. Those with even $m$ generate, via the relation $\lambda_{m}+\alpha=\mu_{m}$, just $n$ different roots $\lambda_{m}$ to (14).

We begin to accomplish this plan by noting that the set of $\mu$ satisfying equation (20) with $m=0$ is the real semi-axis $(0,+\infty)$ containing a single point satisfying (19), namely $\mu_{0}=1+\alpha$.

Similarly, the set of $\mu$ satisfying equation (20) with $m=n$ is the real unbounded interval $(-\infty, 1-n)$ containing a single point satisfying (19), namely $\mu_{n}=\alpha-n$.

Now consider the cases $0<m<n$ and the upper complex half-plane. For any $\omega>0$, the smooth function

$$
\phi_{\omega}(r)=\sum_{j=0}^{n-1} \arg (r+\omega i+j)=\sum_{j=0}^{n-1} \operatorname{arccot} \frac{r+j}{\omega}
$$

monotonically decreases from $n \pi$ to 0 as $r$ increases from $-\infty$ to $+\infty$. So, for any $\omega>0$ and $b \in(0, n \pi)$, there exists a unique value $r$ such that $\phi_{\omega}(r)=b$. Due to the inequality $\frac{d \phi_{\omega}}{d r}(r)<0$, the implicit function theorem provides the existence of the smooth functions $r_{m}(\omega)$ satisfying $\phi_{\omega}\left(r_{m}(\omega)\right)=m \pi$.

Note that if $r \leq-m$, then $r+j<0$ for all $j<m$ and $r+m \leq 0$. Hence,

$$
\varliminf_{\omega \rightarrow+0} \sum_{j=0}^{n-1} \operatorname{arccot} \frac{r+j}{\omega} \geq \varliminf_{\omega \rightarrow+0} \sum_{j=0}^{m-1} \operatorname{arccot} \frac{r+j}{\omega}+\varliminf_{\omega \rightarrow+0}^{\lim } \operatorname{arccot} \frac{0}{\omega}=m \pi+\frac{\pi}{2}>m \pi
$$

and such $r$ cannot be the value of $r_{m}(\omega)$ for sufficiently small $\omega>0$. 
Similarly, if $r \geq 1-m$, then $r+j>0$ for all $j>m-1$ and $r+m-1 \geq 0$. Hence,

$$
\begin{aligned}
\varlimsup_{\omega \rightarrow+0} \sum_{j=0}^{n-1} \operatorname{arccot} \frac{r+j}{\omega} & \leq \varlimsup_{\omega \rightarrow+0} \sum_{j=0}^{m-2} \operatorname{arccot} \frac{r+j}{\omega}+\frac{\pi}{2}+\varlimsup_{\omega \rightarrow+0} \sum_{j=m}^{n-1} \operatorname{arccot} \frac{r+j}{\omega} \\
& \leq(m-1) \pi+\frac{\pi}{2}+0<m \pi
\end{aligned}
$$

and such $r$ cannot be the value of $r_{m}(\omega)$ for sufficiently small $\omega>0$.

So, if $\omega>0$ is sufficiently small, then $r_{m}(\omega)$ satisfies the inequality $-m<r_{m}(\omega)<1-m$ and thereby is negative.

Consider the product $\prod_{j=0}^{n-1}\left|r_{m}(\omega)+\omega i+j\right|$ with $0<m<n$ and investigate its behavior for small $\omega>0$.

If $j \geq m$, then for sufficiently small $\omega>0$, we have $\left|r_{m}(\omega)+j\right|=r_{m}(\omega)+j<j$ and

$$
\prod_{j=m}^{n-1}\left|r_{m}(\omega)+j\right| \leq \prod_{j=m}^{n-1} j<\prod_{j=m}^{n-1}(1+j)
$$

If $j \leq m-1$, then for sufficiently small $\omega>0$, we have $\left|r_{m}(\omega)+j\right|=-r_{m}(\omega)-j<m-j=$ $1+(m-1-j)$

$$
\prod_{j=0}^{m-1}\left|r_{m}(\omega)+j\right| \leq \prod_{j=0}^{m-1}|1+(m-1-j)|=\prod_{J=0}^{m-1}(1+J), \quad J=m-1-j .
$$

Combining (21) and (22), we obtain, for sufficiently small $\omega>0$,

$$
\prod_{j=0}^{n-1}\left|r_{m}(\omega)+j\right|<\prod_{j=0}^{n-1}(1+j)
$$

and

$$
\prod_{j=0}^{n-1}\left|r_{m}(\omega)+\omega i+j\right|<\prod_{j=0}^{n-1}(1+\alpha+j)
$$

As for large $\omega$, the left-hand side of the above inequality evidently tends to $+\infty$ as $\omega \rightarrow$ $+\infty$ and hence is greater than its right-hand side for sufficiently large $\omega$. By continuity there exists $\omega_{m}>0$ such that

$$
\prod_{j=0}^{n-1}\left|r_{m}\left(\omega_{m}\right)+\omega_{m} i+j\right|=\prod_{j=0}^{n-1}(1+\alpha+j)
$$

Thus, we can take $\mu_{m}=r_{m}\left(\omega_{m}\right)+\omega_{m} i \in \mathbb{C}$ to satisfy (19) and (20) for $0<m<n$. For $-n<m<0$, we can take the conjugates $\mu_{m}=\overline{\mu_{-m}}$. Thus, the existence of all $\mu_{m}$ needed is proved. This completes the proof of Proposition 2 . 
Lemma 8 If $12 \leq n \leq 14, \alpha>0$, and $q>0$ satisfy the polynomial equation

$$
\prod_{j=0}^{n-1}\left((\alpha+j)^{2}+q^{2}\right)=\prod_{j=0}^{n-1}(\alpha+j+1)^{2},
$$

then $2 \alpha+4<q^{2}<3 \alpha+5$.

Proof It can be proved in the same way for all $n$ mentioned. We show this for $n=12$.

First, compute the right-hand side of the equation:

$$
\begin{aligned}
\prod_{j=0}^{11}(\alpha & +j+1)^{2} \\
= & \alpha^{24}+156 \alpha^{23}+11,518 \alpha^{22}+535,392 \alpha^{21}+17,581,135 \alpha^{20} \\
& +433,823,676 \alpha^{19}+8,353,410,208 \alpha^{18}+128,665,048,512 \alpha^{17} \\
& +1,612,229,817,055 \alpha^{16}+16,625,859,652,116 \alpha^{15}+142,196,061,481,318 \alpha^{14} \\
& +1,013,438,536,648,512 \alpha^{13}+6,032,418,472,347,265 \alpha^{12} \\
& +29,989,851,619,249,236 \alpha^{11} \\
& +124,253,074,219,885,468 \alpha^{10}+427,135,043,298,835,872 \alpha^{9} \\
& +1,209,806,045,835,003,760 \alpha^{8}+2,795,060,589,044,133,696 \alpha^{7} \\
& +5,194,030,186,679,450,688 \alpha^{6}+7,613,724,634,416,755,712 \alpha^{5} \\
& +8,564,233,279,835,510,784 \alpha^{4}+7,096,936,674,284,421,120 \alpha^{3} \\
& +4,059,952,667,309,260,800 \alpha^{2}+1,424,017,035,657,216,000 \alpha \\
& +229,442,532,802,560,000
\end{aligned}
$$

Now, estimate the left-hand side supposing $q^{2} \geq 3 \alpha+5>0$ :

$$
\begin{aligned}
& \prod_{j=0}^{11}\left((\alpha+j)^{2}+q^{2}\right) \\
& \geq \prod_{j=0}^{11}\left((\alpha+j)^{2}+3 \alpha+5\right) \\
& \geq \alpha^{24}+168 \alpha^{23}+13,216 \alpha^{22}+647,658 \alpha^{21}+22,191,136 \alpha^{20} \\
& \quad+565,650,624 \alpha^{19}+11,143,609,279 \alpha^{18}+174,022,752,156 \alpha^{17} \\
& \quad+2,192,303,359,180 \alpha^{16}+22,557,120,652,044 \alpha^{15}+191,221,185,335,728 \alpha^{14} \\
& \quad+1,343,463,278,373,840 \alpha^{13}+7,851,135,965,424,751 \alpha^{12} \\
& \quad+38,226,775,470,470,448 \alpha^{11} \\
& \quad+155,030,143,411,290,136 \alpha^{10}+522,520,458,095,057,994 \alpha^{9} \\
& \quad+1,457,064,439,886,002,624 \alpha^{8}+3,337,255,633,900,992,816 \alpha^{7}
\end{aligned}
$$




$$
\begin{aligned}
& +6,209,925,089,367,687,345 \alpha^{6}+9,237,499,888,429,090,764 \alpha^{5} \\
& +10,723,421,856,201,549,372 \alpha^{4}+9,360,016,963,404,522,912 \alpha^{3} \\
& +5,777,193,048,791,013,360 \alpha^{2}+2,247,088,906,508,241,600 \alpha \\
& +413,920,896,501,672,000
\end{aligned}
$$

The difference of this polynomial and the previous one is equal to

$$
\begin{aligned}
\prod_{j=0}^{11}\left((\alpha+j)^{2}+3 \alpha+5\right)-\prod_{j=0}^{11}(\alpha+j+1)^{2} \\
=12 \alpha^{23}+1,698 \alpha^{22}+112,266 \alpha^{21}+4,610,001 \alpha^{20}+131,826,948 \alpha^{19} \\
\quad+2,790,199,071 \alpha^{18}+45,357,703,644 \alpha^{17}+580,073,542,125 \alpha^{16} \\
\quad+5,931,260,999,928 \alpha^{15}+49,025,123,854,410 \alpha^{14}+330,024,741,725,328 \alpha^{13} \\
\quad+1,818,717,493,077,486 \alpha^{12}+8,236,923,851,221,212 \alpha^{11} \\
\quad+30,777,069,191,404,668 \alpha^{10}+95,385,414,796,222,122 \alpha^{9} \\
\quad+247,258,394,050,998,864 \alpha^{8}+542,195,044,856,859,120 \alpha^{7} \\
+1,015,894,902,688,236,657 \alpha^{6}+1,623,775,254,012,335,052 \alpha^{5} \\
+ \\
+2,159,188,576,366,038,588 \alpha^{4}+2,263,080,289,120,101,792 \alpha^{3} \\
+1,717,240,381,481,752,560 \alpha^{2}+823,071,870,851,025,600 \alpha \\
+184,478,363,699,112,000
\end{aligned}
$$

which is positive for any $\alpha \geq 0$. This shows that the polynomial equation cannot be satisfied by $\alpha>0$ and $q>0$ with $q^{2} \geq 3 \alpha+5$.

In the same way, compute

$$
\begin{aligned}
\prod_{j=0}^{11}(\alpha & +j+1)^{2}-\prod_{j=0}^{11}\left((\alpha+j)^{2}+2 \alpha+4\right) \\
= & 96 \alpha^{22}+13,156 \alpha^{21}+844,624 \alpha^{20}+33,778,316 \alpha^{19}+943,838,852 \alpha^{18} \\
& +19,590,096,240 \alpha^{17}+313,464,915,984 \alpha^{16}+3,960,996,926,744 \alpha^{15} \\
& +40,162,617,066,616 \alpha^{14}+330,203,929,721,796 \alpha^{13} \\
& +2,215,299,128,334,800 \alpha^{12} \\
& +12,163,303,361,220,828 \alpha^{11}+54,651,209,110,677,476 \alpha^{10} \\
& +200,323,721,839,107,240 \alpha^{9}+595,229,721,350,941,648 \alpha^{8} \\
& +1,419,051,246,703,474,880 \alpha^{7}+2,673,079,829,956,829,568 \alpha^{6} \\
& +3,889,993,689,940,050,432 \alpha^{5}+4,228,750,706,659,177,984 \alpha^{4} \\
& +3,257,831,645,648,401,920 \alpha^{3}+1,625,109,784,526,284,800 \alpha^{2} \\
& +437,271,322,981,376,000 \alpha+37,266,873,282,560,000 .
\end{aligned}
$$


Hence, $\prod_{j=0}^{11}(\alpha+j+1)^{2}>\prod_{j=0}^{11}\left((\alpha+j)^{2}+q^{2}\right)$ if $2 \alpha+4 \geq q^{2}$.

This contradiction yields $2 \alpha+4<q^{2}<3 \alpha+5$. So, Lemma 8 is proved.

The condition $\operatorname{Re} \frac{d \lambda_{\alpha}}{d \alpha}(\tilde{\alpha}) \neq 0$ needed for the Hopf theorem, expressed explicitly by means of the implicit function theorem, looks like

$$
\begin{aligned}
& {\left[\sum_{j=0}^{n-1} \frac{\alpha+j}{q^{2}+(\alpha+j)^{2}}\right]^{2}+\left[\sum_{j=0}^{n-1} \frac{q}{q^{2}+(\alpha+j)^{2}}\right]^{2}} \\
& \quad \neq \sum_{j=0}^{n-1} \frac{\alpha+j}{q^{2}+(\alpha+j)^{2}} \sum_{j=0}^{n-1} \frac{1}{1+\alpha+j} .
\end{aligned}
$$

Lemma 9 If $12 \leq n \leq 14, \alpha>0$ and $0<q^{2}<3 \alpha+5$, then

$$
\begin{gathered}
{\left[\sum_{j=0}^{n-1} \frac{\alpha+j}{q^{2}+(\alpha+j)^{2}}\right]^{2}+\left[\sum_{j=0}^{n-1} \frac{q}{q^{2}+(\alpha+j)^{2}}\right]^{2}} \\
>\sum_{j=0}^{n-1} \frac{\alpha+j}{q^{2}+(\alpha+j)^{2}} \sum_{j=0}^{n-1} \frac{1}{1+\alpha+j} .
\end{gathered}
$$

Proof Hereafter all sums and products with no limits indicated are over $j=0,1, \ldots, n-1$.

Multiplying inequality (23) by $U_{*}=\prod(1+\alpha+j)$ and then twice by $V_{*}=\prod\left[q^{2}+(\alpha+j)^{2}\right]$, we obtain the following equivalent inequality provided $\alpha>0$ :

$$
U_{*}\left[\left(\sum(\alpha+j) V_{j}\right)^{2}+q^{2}\left(\sum V_{j}\right)^{2}\right]>V_{*} \sum(\alpha+j) V_{j} \sum U_{j}
$$

with the polynomials $U_{j}=\frac{U_{*}}{1+\alpha+j}$ and $V_{j}=\frac{V_{*}}{q^{2}+(\alpha+j)^{2}}$.

Put $q^{2}=\frac{3 \alpha+5}{1+w}, w>0$. Substituting this into inequality (24) and multiplying the result by $(1+w)^{2 n-1}$, we obtain another equivalent one:

$$
\begin{aligned}
U_{*}[ & \left.(1+w)\left(\sum(\alpha+j) P_{j}\right)^{2}+(3 \alpha+5)\left(\sum P_{j}\right)^{2}\right] \\
& >P_{*} \cdot \sum(\alpha+j) P_{j} \cdot \sum U_{j}
\end{aligned}
$$

with $P_{*}=\prod\left[3 \alpha+5+(1+w)(\alpha+j)^{2}\right]$ and $P_{j}=\frac{P_{*}}{3 \alpha+5+(1+w)(\alpha+j)^{2}}$.

Both sides of inequality (25) are polynomials of $\alpha$ and $w$ with non-negative integer coefficients. So, they can be computed exactly, with no rounding. This rather cumbersome computation gives the following result for the difference of the left- and right-hand sides of (25) expressed as

$$
\begin{gathered}
U_{*}\left[(1+w)\left(\sum(\alpha+j) P_{j}\right)^{2}+(3 \alpha+5)\left(\sum P_{j}\right)^{2}\right] \\
-P_{*} \sum(\alpha+j) P_{j} \sum U_{j}=\sum_{j=0}^{5 n-2} \Delta_{j} \alpha^{j}
\end{gathered}
$$

with polynomials $\Delta_{j} \in \mathbb{R}[w]$. Straightforward though very cumbersome calculations show that $\Delta_{5 n-2}=0$, and all other $\Delta_{j}$ in (26) are polynomials with positive coefficients.

This completes the proof of Lemma 9. 
To apply the Hopf bifurcation theorem, we need to check that equation (14) cannot have more than a single pair of imaginary conjugated roots. It can be easily obtained by considering equation (16).

Now, the Hopf bifurcation theorem and the lemmas proved provide, for $n=12,13,14$, the existence of a family $\alpha_{\varepsilon}>0$ such that equation (14) with $\alpha=\alpha_{0}$ has imaginary roots $\lambda= \pm q i$ and for sufficiently small $\varepsilon$, system (13) with $\alpha=\alpha_{\varepsilon}$ has a periodic solution $V_{\varepsilon}(t)$ with period $T_{\varepsilon} \rightarrow T=\frac{2 \pi}{q}$ as $\varepsilon \rightarrow 0$. In particular, the coordinate $V_{\varepsilon, 0}(t)=v(t)$ of the vector $V_{\varepsilon}(t)$ is also a periodic function with the same period. Then, taking into account (9), we obtain

$$
y(x)=\left(C+v\left(-\ln \left(x^{*}-x\right)\right)\right)\left(x^{*}-x\right)^{-\alpha} .
$$

Put $h(s)=C+v(-s)$, which is a non-constant continuous periodic and positive for sufficiently small $\varepsilon$ function and obtain the required equality

$$
y(x)=\left(x^{*}-x\right)^{-\alpha} h\left(\ln \left(x^{*}-x\right)\right) .
$$

In the similar way, we obtain the related expressions for $y^{(j)}(x), j=0,1, \ldots, n-1$.

Theorem 4 is proved.

\section{Conclusions, concluding remarks and open problems}

1. Computer calculations give approximate values of $\alpha$ providing equation (14) to have a pure imaginary root $\lambda$. They are, with corresponding values of $k$, as follows:

$$
\begin{aligned}
& \text { if } n=12 \text {, then } \alpha \approx 0.56, k \approx 22.4 ; \\
& \text { if } n=13 \text {, then } \alpha \approx 1.44, k \approx 10.0 ; \\
& \text { if } n=14 \text {, then } \alpha \approx 2.37, k \approx 6.9 \text {. }
\end{aligned}
$$

2. Note that equation (14) has no pure imaginary roots if $n \leq 11$. So, the Hopf bifurcation theorem cannot be applied, but it does not follow that Theorem 4 cannot be proved for some $n<12$.

3. Equation (5) with $n=3$ has solutions of type (6) with oscillatory $h$ (see $[3,5]$ ).

4. If $n \geq 15$, then the inequality needed for the Hopf bifurcation theorem $\operatorname{Re} \frac{d \lambda_{\alpha}}{d \alpha}(\tilde{\alpha}) \neq 0$ cannot be proved in the same way because the estimate $q^{2}<3 \alpha+5$ does not hold.

\section{Competing interests}

The author declares that she has no competing interests.

\section{Acknowledgements}

The research was supported by RFBR (grant 11-01-00989).

Received: 1 March 2013 Accepted: 21 June 2013 Published: 23 July 2013

\section{References}

1. Kiguradze, IT, Chanturia, TA: Asymptotic Properties of Solutions of Nonautonomous Ordinary Differential Equations. Kluwer Academic, Dordrecht (1993)

2. Astashova, IV: Asymptotic behavior of solutions of certain nonlinear differential equations. In: Reports of Extended Session of a Seminar of the I. N. Vekua Institute of Applied Mathematics, vol. 1(3), pp. 9-11. Tbilis. Gos. Univ., Tbilisi (1985) (Russian)

3. Astashova, IV: Qualitative properties of solutions to quasilinear ordinary differential equations. In: Astashova, IV (ed.) Qualitative Properties of Solutions to Differential Equations and Related Topics of Spectral Analysis, scientific edition, pp. 22-290. UNITY-DANA, Moscow (2012) (Russian)

4. Kozlov, VA: On Kneser solutions of higher order nonlinear ordinary differential equations. Ark. Mat. 37(2), 305-322 (1999) 
5. Astashova, IV: Application of dynamical systems to the study of asymptotic properties of solutions to nonlinear higher-order differential equations. J. Math. Sci. 126(5), 1361-1391 (2005)

6. Marsden, JE, McCracken, M: The Hopf Bifurcation and Its Applications. Springer, Berlin (1976). XIII

7. Astashova, IV, Vyun, SA: On positive solutions with non-power asymptotic behavior to Emden-Fowler type twelfth order differential equation. Differ. Equ. 48(11), 1568-1569 (2012) (Russian)

doi:10.1186/1687-1847-2013-220

Cite this article as: Astashova: On power and non-power asymptotic behavior of positive solutions to Emden-Fowler type higher-order equations. Advances in Difference Equations 2013 2013:220.

Submit your manuscript to a SpringerOpen ${ }^{\circ}$ journal and benefit from:

- Convenient online submission

- Rigorous peer review

- Immediate publication on acceptance

- Open access: articles freely available online

- High visibility within the field

- Retaining the copyright to your article

Submit your next manuscript at $>$ springeropen.com 